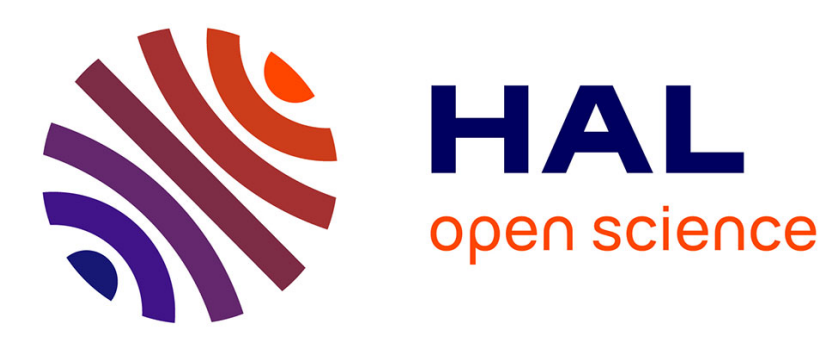

\title{
Dissipation Assessments During Dynamic Very High Cycle Fatigue Tests
}

\author{
Antoine Blanche, André Chrysochoos, Nicolas Ranc, Véronique Favier
}

\section{To cite this version:}

Antoine Blanche, André Chrysochoos, Nicolas Ranc, Véronique Favier. Dissipation Assessments During Dynamic Very High Cycle Fatigue Tests. Experimental Mechanics, 2015, 55, pp.699-709. 10.1007/s11340-014-9857-3 . hal-01178226

\section{HAL Id: hal-01178226 \\ https://hal.science/hal-01178226}

Submitted on 17 Jul 2015

HAL is a multi-disciplinary open access archive for the deposit and dissemination of scientific research documents, whether they are published or not. The documents may come from teaching and research institutions in France or abroad, or from public or private research centers.
L'archive ouverte pluridisciplinaire HAL, est destinée au dépôt et à la diffusion de documents scientifiques de niveau recherche, publiés ou non, émanant des établissements d'enseignement et de recherche français ou étrangers, des laboratoires publics ou privés. 


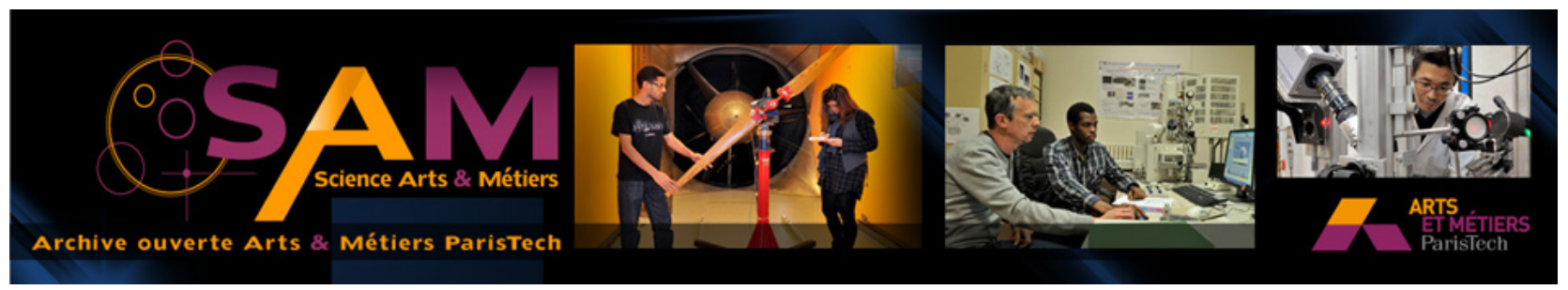

Science Arts \& Métiers (SAM)

is an open access repository that collects the work of Arts et Métiers ParisTech researchers and makes it freely available over the web where possible.

This is an author-deposited version published in: http://sam.ensam.eu

Handle ID: .http://hdl.handle.net/10985/9826

\section{To cite this version :}

Antoine BLANCHE, A CHRYSOCHOOS, Nicolas RANC, Véronique FAVIER - Dissipation Assessments During Dynamic Very High Cycle Fatigue Tests - Experimental Mechanics - Vol. 55, p.699-709- 2015 


\title{
Dissipation Assessments During Dynamic Very High Cycle Fatigue Tests
}

\author{
A. Blanche $\cdot$ A. Chrysochoos $・$ N. Ranc $\cdot$ V. Favier
}

\begin{abstract}
This paper presents an experimental device developed to detect and estimate dissipated energy during very high cycle fatigue tests (VHCF) at high loading frequency $(20 \mathrm{kHz})$ and low stress (i.e. far below the yield stress). Intrinsic dissipation is computed using local expressions of the heat diffusion equation and thermal data fields provided by an infrared focal plane array camera. The results obtained from tests performed on pure copper specimens show that dissipated energy exists whatever the attainable stress range and show that the dissipated energy rate is not constant throughout the test. Both findings are respectively incompatible with the concepts of fatigue limit based on elastic shakedown or on stabilized cyclic state associated with the mechanical hysteresis loop (viscoplastic shakedown).
\end{abstract}

Keywords Intrinsic dissipation · Ultrasonic fatigue · Infrared thermography $\cdot$ Fatigue limit $\cdot$ Hysteresis loop

\footnotetext{
A. Blanche $(\bowtie) \cdot$ A. Chrysochoos

Laboratoire de Mécanique et Génie Civil (LMGC), Université

Montpellier 2, CNRS, Place Eugène Bataillon, 34090 Montpellier, France

e-mail: antoine.blanche@univ-montp2.fr

A. Chrysochoos

e-mail: andre.chrysochoos@univ-montp2.fr

A. Blanche $\cdot$ N. Ranc $\cdot$ V. Favier

Laboratoire Procédés et Ingénierie en Mécanique et Matériaux

(PIMM), UMR CNRS 8006, Arts et Métiers Paristech, 151

boulevard de l'Hôpital, 75013 Paris, France

N. Ranc

e-mail: nicolas.ranc@ensam.eu

V. Favier

e-mail: veronique.favier@ensam.eu
}

\section{Introduction}

The existence of a fatigue limit is still controversial [1-3]. The fatigue limit is usually estimated from curves plotted for the stress vs. the number of cycles (SN). It is generally acknowledged, even by the industry standards, that above $10^{7}$ cycles a more or less horizontal asymptote on this curve determines the fatigue limit. It is also more or less implicitly assumed that a viscoelastic or elastoplastic shakedown would occur below this limit, with the fatigue lifespan thus theoretically becoming infinite.

Nevertheless, previous studies [3] have shown that some materials can break in the VHCF regime below the traditional fatigue limit. This highlights the importance of performing fatigue tests at a very high number of cycles. The main difficulty, in this case, is to reach more than $10^{8}-10^{9}$ cycles in reasonable time. Many VHCF testing machines have been developed to reduce the test duration [4]. They involve performing fatigue tests at high loading frequencies (e.g. more than $1000 \mathrm{~Hz}$ ). For such frequencies, the acceleration quantities are generally no longer negligible and the fatigue test must then be considered as a dynamic test. In this situation, it is thus necessary to check the stress pattern over the sample gage part and analyze the possible influence of self-heating and the loading frequency on the behavior of the tested material [5-8].

Regarding the modeling of the fatigue limits and lifespan, numerous mechanical-based uniaxial approaches have proposed phenomenological links between the hysteresis loop characteristics (e.g. Miner-Wöhler's law, Haig's diagram, Goodman-Soderberg's rules, etc.) and the fatigue limit and/ or the number of cycles to failure [9-12]. Multiaxial fatigue criteria (e.g. Crossland, Sines, Dang-Van, etc.) starting from phenomenological or microstructural considerations were also proposed. Note that all of these approaches assumed a rapid stabilization of the hysteresis loop size for constant loading conditions. All of these design tools have been, and still are, 
beneficially used in numerous industrial applications to estimate the fatigue strength of mechanical parts. However, the experimental identification of such criteria for each particular case is very time consuming.

This is the reason why alternative methods were recently proposed. Some of them are based on the thermal response of material subjected to fatigue loadings. More particularly, they analyze self-heating during stepwise loading fatigue tests involving cycle blocks with increasing stress ranges. The goal is to find a change in the heating regime empirically related to the fatigue limit of the material [13-22]. Note that, to determine the fatigue lifespan, these approaches assume that the material has a certain mechanical energy reserve to dissipate before rupture. The number of cycles to failure is computed by estimating the area of the stabilized hysteresis loop, while assuming that the energy mechanically lost per cycle is entirely dissipated. It is much faster to obtain self-heating diagrams than it is to plot Wölher curves. This explains the keen interest that some R\&D partners have for thermal methods.

The following experimental work is based on calorimetric analysis of the material response during high frequency loading. To our knowledge, this is the first attempt to assess dissipation fields during dynamic fatigue tests. The dissipation estimates are very sensitive because of the dynamic loading, the complex thermal situation, and, as usual, the regularizing effect of heat diffusion. However, these calorimetric data are essential safeguards to study the relevance of the fatigue limit concept, determine the nature of dissipative effects and check their constancy over the test, which is essential for a stabilized cyclic state. The fatigue limit and stabilized mechanical hysteresis loop concepts will be assessed from a thermodynamic standpoint, with elastic shakedown corresponding to a nondissipative process, while plastic shakedown reflects a steady dissipative process. The layout of the paper is as follows:

- The experimental setup is first introduced. It mainly involves an ultrasonic fatigue testing machine with a loading frequency of about $20 \mathrm{kHz}$ and an infrared workstation.

- Thermal diffusion models and image processing methods used to compute the intrinsic dissipation patterns are then presented.

- Experimental results obtained on pure copper are finally shown and analyzed. Their consequences on some fatigue modeling foundations are finally discussed.

\section{Experimental Setup}

The experimental setup involves an ultrasonic fatigue testing machine with a loading frequency 1000 times higher than conventional frequencies (here, $20 \mathrm{kHz}$ ). There are therefore advantages reaching the VHCF regimes within a reasonable time. Moreover, high frequency loading increases the dissipation intensity and thus generates large temperature variations that are easily detectable even by standard thermal measurement devices. The first ultrasonic fatigue machine was constructed by Mason in 1950 [23]. The ultrasonic actuators are designed assuming an elastic behavior of the material to test and the specimen movements are supposed to be free steadystate vibrations. The experimental setup essentially includes the three components $[24,25]$ shown in Fig. 1a:

- a piezoelectric transducer, which transforms an electrical signal into a displacement

- a horn, which amplifies this displacement

- a specimen screwed on the horn and free of stress at its bottom extremity.

This dynamic loading system and the "elastic" specimen are designed, using the wave equation, to have the first longitudinal eigen vibration mode at $20 \mathrm{kHz}$. The load ratio is $R_{\sigma}=\sigma_{\min } / \sigma_{\max }=-1$. This system is calibrated with a laser extensometer to obtain the relation between the displacement variation on the horn edge and the input electrical signal. Assuming an elastic behavior it is then possible to compute the steady state stress distribution along the specimen length. Figure $1 \mathrm{~b}$ shows a basic sketch of the stress range pattern along the gage part of the specimen [25]. As the influence of the stress gradient on the $\mathrm{SN}$ curves is sometimes reported [26], its influence on the dissipation profiles will be discussed in the "Results and Comments" section.

\section{Fatigue Tests}

The specimens used in this work are hourglass shaped plate specimens in commercial polycrystalline copper $\mathrm{CuOF}$ $99.95 \%$ (supplied by Griset). The Young's modulus is $130 \mathrm{GPa}$. All specimens are systematically annealed at $250{ }^{\circ} \mathrm{C}$ for $60 \mathrm{~min}$ to relieve the residual stresses in the bulk specimen. The resulting mean grain size is then approximately $50 \mu \mathrm{m}$. The fatigue strength amplitude is about $93 \mathrm{MPa}\left(\mathrm{R}_{\sigma}=\right.$ -1 ) at $10^{10}$ cycles [27] and the tensile yield stress at $0.2 \%$ is $87 \mathrm{MPa}$ for a ultimate tensile strength of about $230 \mathrm{MPa}$ [28]. After mechanical and electrolytic polishing, the specimen surfaces are mirror polished with low residual stress [28].

The fatigue tests consist of performing $10^{6}$ to $10^{9}$ cycles at different stress ranges (from $\Delta \sigma / 2=20 \mathrm{MPa}$ to $90 \mathrm{MPa}$ ) for stress ratio $R_{\sigma}=-1$ and at loading frequency $f_{\mathrm{L}}=20 \mathrm{kHz}$. Each stress range is numerically assessed in the middle crosssection of the specimen $(x=0)$ for each electrical tension applied to the piezoelectric transducer : as previously mentioned, the displacement of the horn is monitored and used as boundary conditions in the model while an isotropic elastic behavior of the tested material has been assumed. However, because of the irreversibility of the fatigue process, whose the 
Fig. 1 (a) Sketch of the piezoelectric system; (b) stress pattern and specimen characteristics (a)

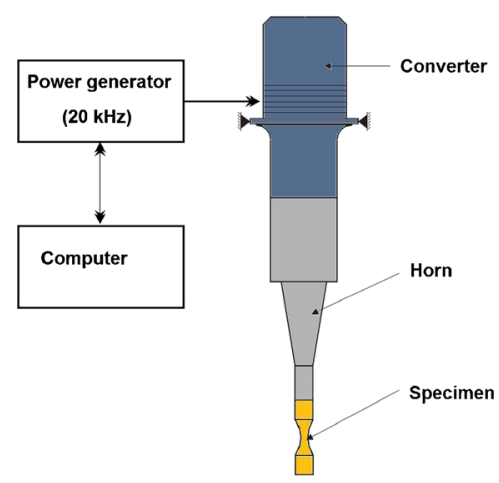

(b)

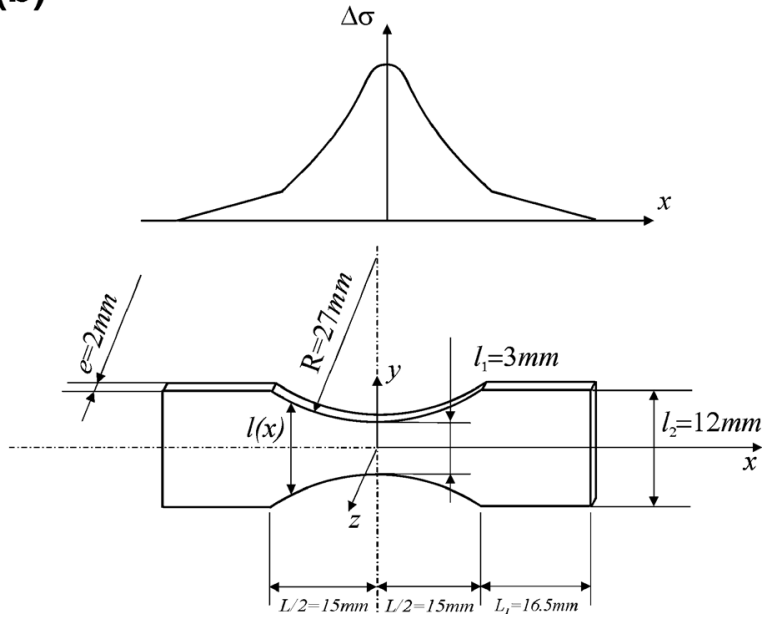

dissipation is the calorimetric signature, VHCF experiments are not really stress-controlled or free steady-state vibration tests.

\section{Camera Calibration}

The infrared focal plane array (IRFPA) camera used in this work (Titanium camera designed by FLIR-CEDIP) is equipped with an InSb $512 \times 640$ element detector. During the test, the camera lens axis is kept fixed and held perpendicular to the surface of the specimen during the tests. An individual pixel calibration of this camera is performed using an extended black body [29]. The calibration is based on a polynomial fitting of the temperature as a function of the signal delivered by each detector element. Here, nonuniformity correction (NUC) and bad pixel replacement (BPR) are no-longer considered. Bad pixels are not considered in the image processing presented hereafter. The specimen surface is painted matte black to have a surface emissivity close to one. It is then possible to measure temperature fields at the specimen surface. Spatial resolution and thermal accuracy are respectively about $0.1 \mathrm{~mm} /$ pixel and $20 \mathrm{mK}$. The frame rate of the IR camera is typically about $10 \mathrm{~Hz}$. Readers interested in these tricky metrological issues can refer to [29].

\section{Heat Equation}

Although ultrasonic fatigue tests are dynamic mechanical processes, they are considered as quasi-static processes from a thermodynamic standpoint (see the statement of the local state axiom in [30]). Consequently, a finite set of variables can be chosen to describe the equilibrium material state. This generally involves the absolute temperature $T$, the (small) strain tensor $\varepsilon$ and a vector $\alpha$ of $N$ internal state variables. Combining the local expressions of the first and second principles of thermodynamics, and assuming the Fourier heat conduction law, the local heat diffusion equation can be written as:

$\rho C \dot{T}-\operatorname{div}(k \operatorname{grad}(T))=d_{1}+s_{\text {the }}+s_{\text {thc }}+r_{\text {ext }}$

where $\rho$ is the mass density, $C=-T \psi{ }_{T T}$ the specific heat, $\psi$ the Helmholtz free energy, $k$ an isotropic conduction tensor. The left hand side of this equation is a differential operator applied to $T$ while its right hand side gathers different heat sources. These are in turn: the intrinsic dissipation $d_{1}=\sigma: \dot{\varepsilon}-\rho \psi,,_{\varepsilon}: \dot{\varepsilon}-\rho \psi,{ }_{\alpha} . \dot{\alpha}$, where $\sigma$ is the Cauchy stress tensor, the thermoelastic source $s_{\text {the }}$, the other possible thermomechanical coupling sources $s_{\text {thc }}$ ( with $s_{\text {the }}+s_{\text {thc }}=\rho T \psi,_{T \varepsilon}: \dot{\varepsilon}+\rho T,_{T \alpha} \cdot \dot{\alpha}$ ) and the external volume heat supply $r_{\text {ext }}$.

The local heat diffusion equation can be simplified assuming the following hypotheses [31, 32]:

- The parameters $\rho$ and $C$ are material constants, independent of the state variables.

- The isotropic conduction tensor $k$ is also a material constant independent of the state variables.

- Thermoelastic effects induced by the thermo-dilatability are the only thermomechanical coupling factors considered hereafter (experiments on pure copper close to room temperature).

- Because of the thermal inertia, the thermal gradients slowly vary during the tests as compared to the characteristic cyclic loading time. Moreover, the mean velocity field vanishes over a cycle for such periodic loading. Consequently, the convective terms of the total time derivative of the temperature were neglected. 
- The external heat supply is time independent. The equilibrium temperature field $T_{0}$ verifies $-k \Delta T_{0}=r_{\text {ext }}$. The temperature variations are noted $\theta=T-T_{0}$.

Under these hypotheses, the local heat diffusion equation is simplified into the following form:

$\rho C \frac{\partial \theta}{\partial t}-k \Delta \theta=d_{1}+s_{\text {the }}$

Taking the loading frequency $(20 \mathrm{kHz})$ and the maximum frame rate of the IR camera $(100 \mathrm{~Hz})$ into account (integration time $=1000 \mu \mathrm{s}=20$ cycles $)$, the thermoelastic source amplitude $s_{\text {the }}$ are here out of reach. Only the mean dissipation per cycle was derived from thermal data using, by construction, data corresponding to averaged temperatures over 20 loading cycles. Moreover, dissipation was estimated by evaluating the left hand side of the heat equation integrating equation (2) over the sample thickness (2D diffusion model) or over the sample cross-section (1D diffusion model). Under the previous hypotheses for thin flat samples (cf. Fig. 1b), it has been shown in [31] that the mean temperature over the thickness (over the cross section) remains close to the corresponding surface temperature given by the IR camera.

\section{D Heat Diffusion Model}

Under some additional hypotheses, it is possible to use an integrated form of the heat equation over the sample thickness to evaluate the depth-wise averaged heat source distribution [33]. These hypotheses are:

- The heat exchanges between front and back specimen surfaces and the surroundings are symmetric and linear with respect to the temperature variation.

- The infrared surface temperatures remain close to the depth-wise averaged temperature field.

The 2D heat diffusion model can finally be formulated as:

$\left.\rho C \frac{\partial \bar{\theta}}{\partial t}+\frac{\bar{\theta}}{\tau^{2 D}}\right)-k\left(\frac{\partial^{2} \bar{\theta}}{\partial \mathrm{x}^{2}}+\frac{\partial^{2} \bar{\theta}}{\partial \mathrm{y}^{2}}\right)=\bar{d}_{1}$

where $\bar{\theta}$ and $\overline{d_{1}}$ respectively represent the depth-wise average distributions of $\theta$ and $d_{1}$. The time constant $\tau^{2 D}=\frac{\rho C e}{2 h}$ characterizes perpendicular heat exchanges between the front and back specimen faces and the surroundings, where $h$ and $e$ are the heat exchange coefficient and the sample thickness, respectively. For the practical use of equation (3), the temperature measured at the specimen surface must remain close to the mean temperature over the cross-section $S$. This assumption is especially relevant since the sources are regularly distributed over $S$ and the Biot number $B_{i}=h l_{c} / k$ is small compared to the unit [34]. The characteristic length $l_{c}$ is commonly defined as the volume of the body divided by its surface area. For the copper specimens, the Biot number is about $10^{-4}$. In practice again, it is also often noted that widthwise heat losses are negligible compared with the length-wise losses $\left(\frac{\partial^{2} \bar{\theta}}{\partial \mathrm{y}^{2}} \ll \frac{\partial^{2} \bar{\theta}}{\partial \mathrm{x}^{2}}\right)$. This result is due to the fact that the testing machine grips act as heat wells for materials with high diffusivity. This is certainly the case of the pure copper studied here, for which the heat diffusion parameter is about $10^{-4} \mathrm{~m}^{2} \cdot \mathrm{s}^{-1}$.

\section{D Heat Diffusion Model}

The heat equation can also be integrated over each cross section area $S(x)=e . l(x)$ to finally obtain a $1 \mathrm{D}$ heat diffusion model [32, 35]:

$\rho C\left(\frac{\partial \overline{\bar{\theta}}}{\partial t}+\frac{\overline{\bar{\theta}}}{\tau^{1 D}}\right)-k\left(\frac{\partial^{2} \overline{\bar{\theta}}}{\partial \mathrm{x}^{2}}+\frac{1 \mathrm{~d} S \partial \overline{\bar{\theta}}}{S \mathrm{dx} \partial \mathrm{x}}\right)=\overline{\bar{d}}_{1}$

where the mean temperature variation over the cross section $\overline{\bar{\theta}}(x, t)$ is identified to the width-wise average temperature given by the IR camera. The time constant $\tau^{1 D}(x)=\frac{\rho C S(x)}{2 h(e+l(x))}=\tau^{2 D} \frac{l(x)}{(e+l(x))} \quad$ characterizes the global lateral heat exchanges by conduction, convection and radiation between the specimen cross-section and the surroundings.

\section{Heat Exchange Coefficient Estimate}

In 2D and 1D diffusion models, it is necessary to estimate a time constant $\left(\tau^{1 D}\right.$ or $\left.\tau^{2 D}\right)$ characterizing local heat losses. This time constant depends on the specimen geometry, the material constant and also the heat exchange coefficient $h$. This coefficient is usually experimentally determined.

This identification is achieved by analyzing thermoprofiles (1D analysis) during a thermal return to equilibrium. In this case, no heat source occurs. All the parameters of the 1D heat diffusion models are known except $\tau^{1 D}(h)$. It is then possible to estimate $h$ using a least-squares method. The best value of $h$ named $h_{\text {opt }}$ minimizes the following function $\mathcal{R}$ :

$$
\left\{\begin{array}{l}
\mathcal{R}(h)=\iint_{\mathcal{L} \times D}\left[\rho C\left(\frac{\partial \overline{\bar{\theta}}}{\partial t}+\frac{\overline{\bar{\theta}}}{\tau^{1 D}(h)}\right)-k\left(\frac{\partial^{2} \overline{\bar{\theta}}}{\partial x^{2}}+\frac{1}{S} \frac{\mathrm{d} S}{\mathrm{~d} x} \frac{\partial \overline{\bar{\theta}}}{\partial x}\right)\right]^{2} \mathrm{~d} x \mathrm{~d} t \\
\left.\frac{\partial \mathcal{R}}{\partial h}\right|_{h=h_{\text {opt }}}=0
\end{array}\right.
$$


where $\mathcal{L}$ and $D$ represent the thermo-profile length and the thermal return duration, respectively. To simplify the presentation, a continuous version of the function to minimize has been written in equation (5). Practically, discretized version of equation (5) is naturally used. This latter requires an evaluation of each differential operator (see section "Heat Source Computation"). As a result, $h_{\text {opt }}$ was found in ranges of 30 $100 \mathrm{~W} \cdot \mathrm{m}^{-2} \cdot \mathrm{K}^{-1}$ according to the experimental conditions. These orders of magnitude show that the heat losses are not only induced by the natural convection but also by the turbulent airflow above the specimen (i.e. forced convection), which is aimed at cooling the piezoelectric converter and then the specimen. In the experimental and simulated results shown hereafter, a heat exchange of about $h_{\text {opt }}=30 \mathrm{~W} \cdot \mathrm{m}^{-2} \cdot \mathrm{K}^{-1}$ was used. However, it is worth noting that the contribution of the out-ofplane heat losses, remained low compared with the longitudinal ones irrespective of the possible value of $h$. In others words, the value of $h$ had little influence on the heat source assessment for this pure copper material and specimen geometry.

A 1D thermal computation can be performed to check the relevance of the heat exchange coefficient estimate. This involves comparing a predicted thermo-profile to an experimental thermo-profile during a thermal return. This predicted thermoprofile is found by solving the following partial differential equation where $\overline{\bar{\theta}}_{\exp }(x, t=0)$ and $\overline{\bar{\theta}}_{\text {exp }}(x= \pm L / 2, t)$ are the experimental initial and Dirichlet's boundary conditions.

$$
\left\{\begin{array}{l}
\rho C\left(\frac{\partial \overline{\bar{\theta}}}{\partial t}+\frac{\overline{\bar{\theta}}}{\tau^{1 D}}\right)-k\left(\frac{\partial^{2} \overline{\bar{\theta}}}{\partial x^{2}}+\frac{1 \mathrm{~d} S \partial \overline{\bar{\theta}}}{S} \frac{\mathrm{dx} \partial x}{\partial x}=0\right. \\
\overline{\bar{\theta}}(x, t=0)=\overline{\bar{\theta}}_{\exp }(x, t=0) \\
\overline{\bar{\theta}}(x= \pm L / 2, t)=\overline{\bar{\theta}}_{\exp }(x= \pm L / 2, t)
\end{array}\right.
$$

Figure 2 shows the different temperature profiles. The results of the comparison of the experimental and predicted

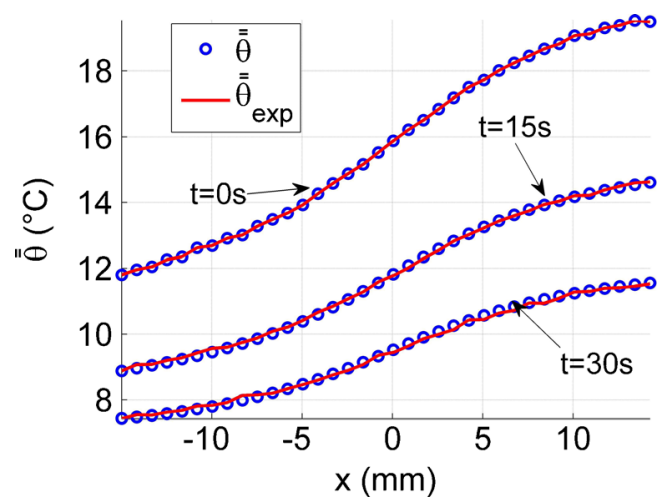

Fig. 2 Measured and predicted temperature profiles during a thermal return

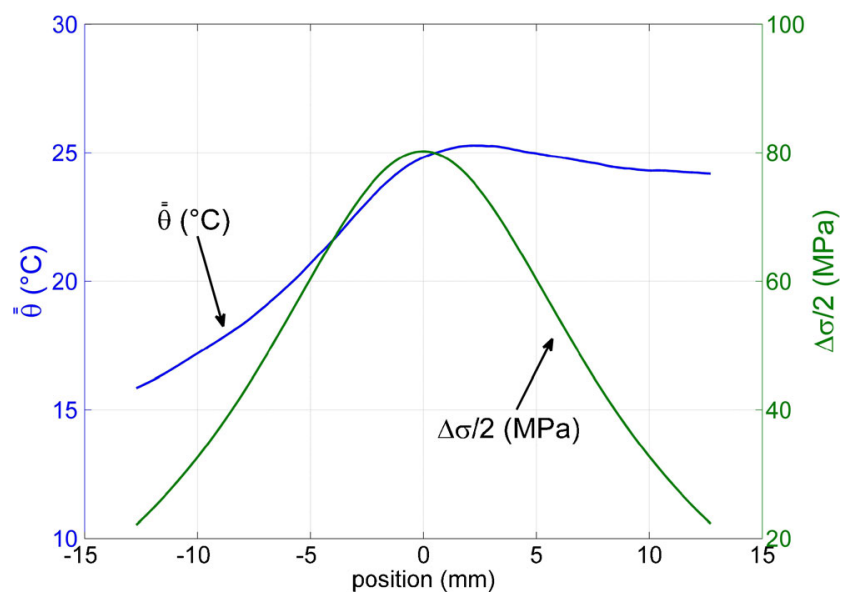

Fig. 3 Typical example of stress amplitude and temperature profiles

results during the thermal return are pretty good, showing that the 1D thermal model is relevant for describing the complex thermal situation. The absolute error is in the same order of magnitude as thermal accuracy of the camera $(20 \mathrm{mK})$.

The determination of $\tau^{1 D}$ immediately leads to an estimate of $\tau^{2 D}$ using the relation $\tau^{2 D}=\frac{e+l(x)}{l(x)} \tau^{1 D}$.

\section{Effects of the Thermal Boundary Conditions}

Figure 3 shows stress amplitude and temperature profiles along the specimen length. Note that the stress distribution is symmetric whereas the temperature profile is not. As already mentioned, this dissymmetry is due to the thermal boundary conditions. One extremity of the sample $(x=-\mathrm{L} / 2)$ is fixed to the horn-converter system cooled by a cold air jet while the sample is totally free on the other side $(x=+\mathrm{L} / 2)$.

Because of this non-symmetry and heat diffusion mechanisms, a high stress level does not systematically correspond to a high temperature. The following section illustrates the critical role played by heat diffusion in the calorimetric analysis.

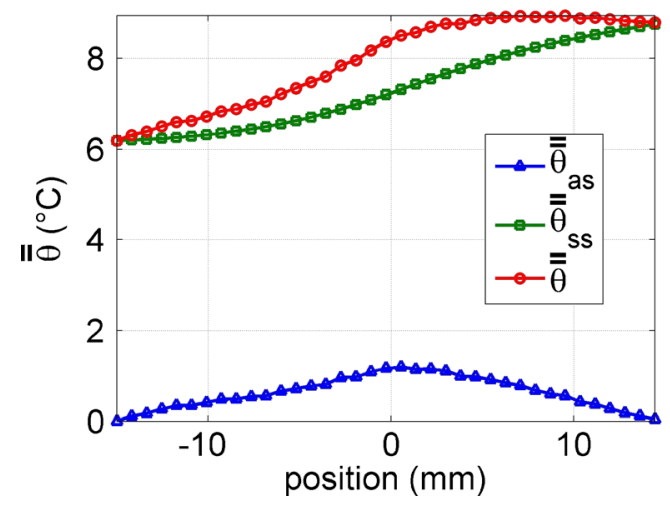

Fig. 4 Temperature decomposition 


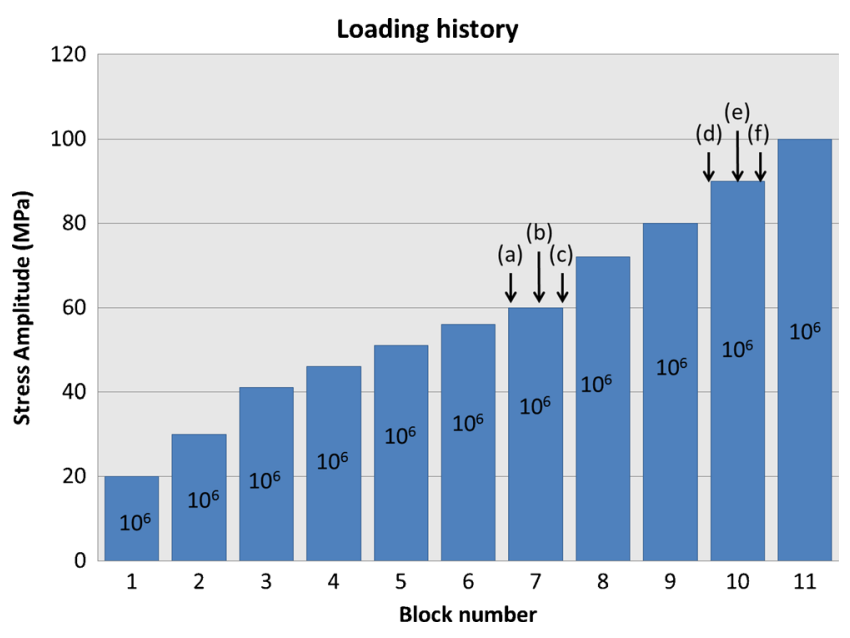

Fig. 5 Basic sketch of the fatigue tests; $a, b, . ., f$ corresponds to times at which the results shown hereafter were captured. Fatigue blocks were made of $10^{6}$ cycles performed at $R_{\sigma}=-1$ for increasing stress amplitudes $(\Delta \sigma / 2)$

The heat equation (equation (4)) is indeed linear with respect to the temperature. Consequently, it is possible to use the superposition principle. We consider hereafter that the experimental temperature profile (see Fig. 4) is the result of two contributions:

$\overline{\bar{\theta}}(x, t)=\overline{\bar{\theta}}_{s s}(x, t)+\overline{\bar{\theta}}_{a s}(x, t)$

- where $\overline{\bar{\theta}}_{s s}$ takes the influence of the boundary conditions into account. This temperature profile represents the temperature profile in the situation where no heat source occurs within the specimen gage part.

- where $\overline{\bar{\theta}}_{\text {as }}$ only takes the heat source distribution into account. This heat sources is naturally unknown in practice. The construction of $\overline{\bar{\theta}}_{\text {as }}$ is sometimes called the lifting of the temperature variation $\overline{\bar{\theta}}$.
The experimental temperature profile is the solution of the following system where $\vartheta_{0}(x)$ and $\vartheta_{ \pm \frac{L}{2}}(t)$ are the initial and Dirichlet boundary conditions, respectively:

$$
\left\{\begin{array}{l}
\rho C\left(\frac{\partial \overline{\bar{\theta}}}{\partial t}+\frac{\overline{\bar{\theta}}}{\tau^{1 D}}\right)-k\left(\frac{\partial^{2} \overline{\bar{\theta}}}{\partial x^{2}}+\frac{1}{S} \frac{\mathrm{d} S \partial \overline{\bar{\theta}}}{\mathrm{dx} \partial x}\right)=s \\
\overline{\bar{\theta}}(x, t=0)=\vartheta_{0}(x) \\
\overline{\bar{\theta}}(x= \pm L / 2, t)=\vartheta_{ \pm \frac{L}{2}}(t)
\end{array}\right.
$$

This system can be split into two systems to compute $\overline{\bar{\theta}}_{\mathrm{ss}}$ and then to derive $\overline{\bar{\theta}}_{\text {as }}=\overline{\bar{\theta}}-\overline{\bar{\theta}}_{\text {ss }}$. This partition is performed to separate the thermal effects induced by complex boundary conditions from those induced by the heat source distribution.

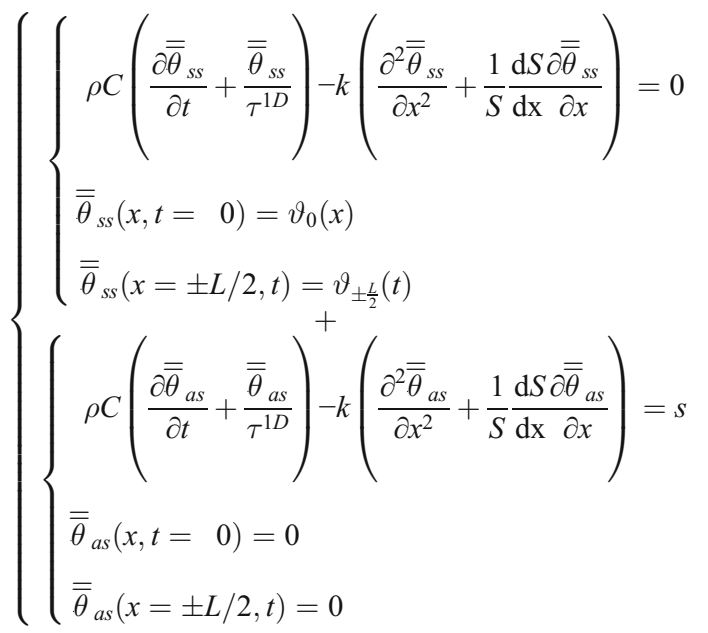

The illustrative example shown in Fig. 4 is extracted from a fatigue test. Note the relative importance of $\overline{\bar{\theta}}_{s s}$ and $\overline{\bar{\theta}}_{a s}$ and the effect of the dissymmetry of the boundary conditions on $\overline{\bar{\theta}}_{s s}$. Concerning the temperature profile $\overline{\bar{\theta}}_{a s}(x, t)$, it
Fig. 6 Time course of the dissipation profiles as a function of the number of cycles (a) $\Delta \sigma / 2=$ $60 \mathrm{MPa}$ (b) $\Delta \sigma / 2=90 \mathrm{MPa}$ (a)

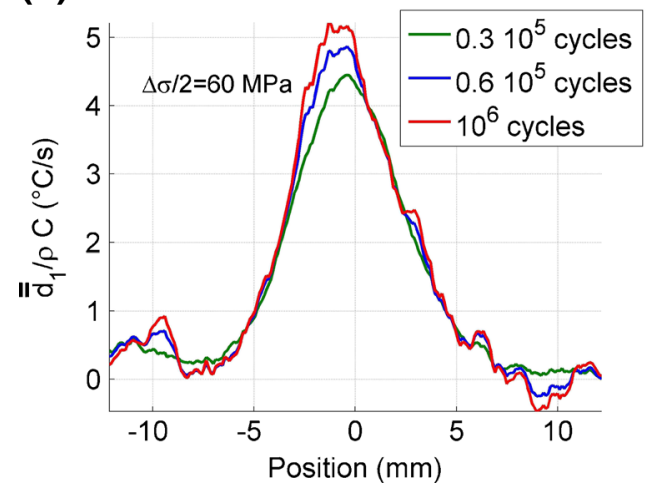

(b)

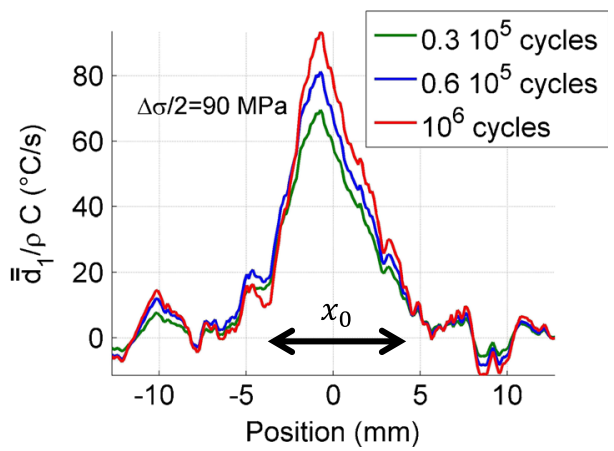




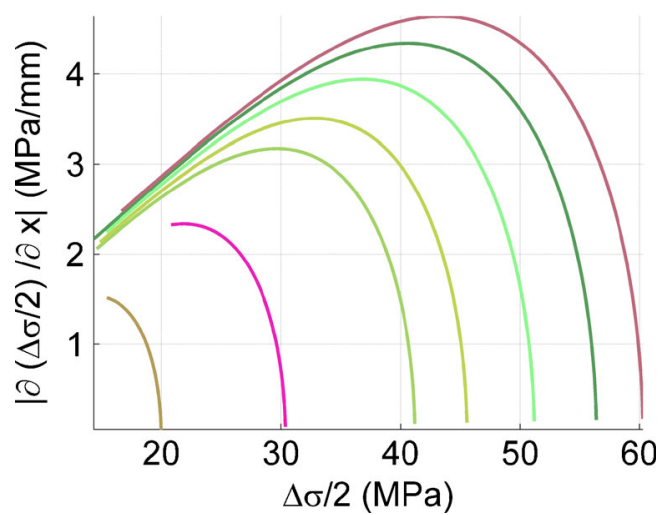

Fig. 7 Stress gradient versus the stress curve

corresponds to the temperature induced by the heat source distribution under homogeneous Dirichlet boundary conditions. This distribution can be more easily correlated to the stress pattern shown in Fig. 3.

\section{Heat Source Computation}

To derive heat sources from thermal images, we usually directly estimate the left hand member of the heat equation using either $\overline{\bar{\theta}}$ or $\overline{\bar{\theta}}_{\text {as }}$ thermal data. Different techniques have been developed and used over the last years [31-33, 35]. In this work, the heat source assessment is based on a local approximation of the temperature field using a least-squares fitting. The approximation function reads:

$\theta_{\text {app }}=P_{1}(x, y) t+P_{2}(x, y)$

where $P_{i}$ are second order polynomials in $x$ and $y$. These polynomials enabled us to compute each partial differential operator of the heat diffusion equation. Hence, when knowing a local polynomial approximation of the temperature fields, it is possible to evaluate the intrinsic dissipation distribution $d_{1}$.

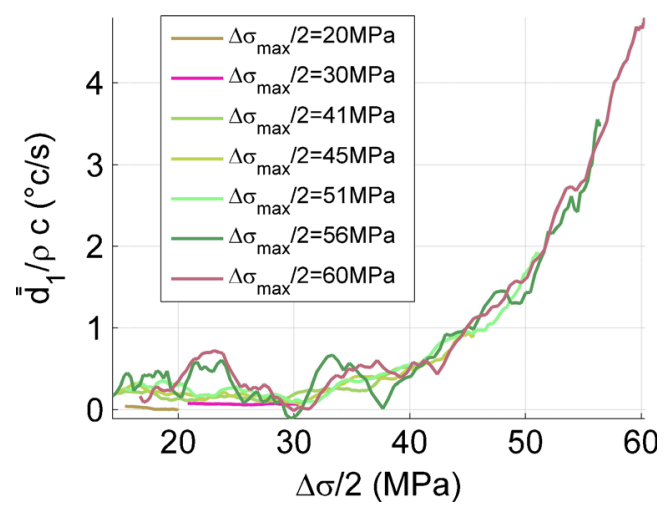

Fig. 8 Mean dissipation over the cross-section versus the stress curve (a)

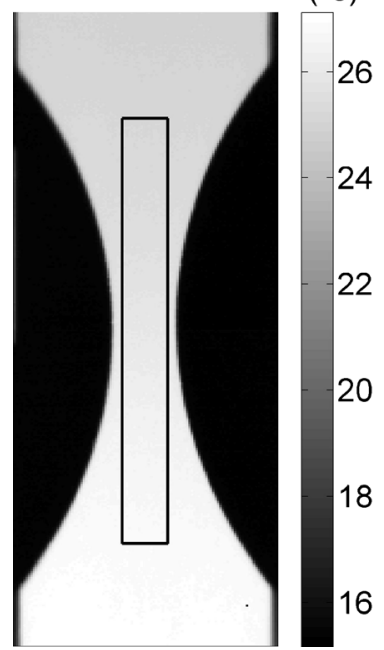

(b)

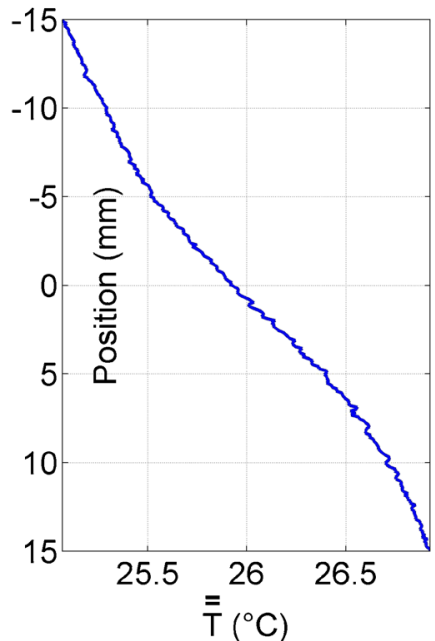

Fig. 9 Examples of (a) temperature field and (b) longitudinal thermoprofile associated with the $2 \mathrm{D}$ and $1 \mathrm{D}$ distributions of dissipation

\section{Results and Comments}

In the following, intrinsic dissipation $d_{1}$ is divided by $\rho C$ in order to have the volume energy rate $\left(\mathrm{W} / \mathrm{m}^{3}\right)$ expressed in $\left({ }^{\circ} \mathrm{C} /\right.$ $\mathrm{s})$. This change of unit facilitates the data interpretation.

\section{First Dissipation Properties}

Tests were conducted as described in Fig. 5. Fatigue tests were periodically stopped at the end of each block of cycles, with the stress amplitude (half of the stress range) increasing from block to block. The stress range was assessed considering the cross-section $\mathrm{x}=0$.

Figures 6(a-b) show intrinsic dissipation profiles along the specimen at 60 and $90 \mathrm{MPa}$ during a $10^{6}$ cycle fatigue test (block \#7: (a) $0.3 \times 10^{5}$ cycles (b) $0.6 \times 10^{5}$ cycles (c) $10^{6}$ cycles and block \#10: (d) $0.3 \times 10^{5}$ cycles (e) $0.6 \times 10^{5}$ cycles (f) $10^{6}$ cycles). We note here that the highest dissipation is close to the center section of the specimen, where the stress intensity is also highest (see Figs. 2 and 4). However, this result was not systematic. Sometimes a high dissipation zone was observed further away from the specimen center. Nevertheless, whatever the localization locus, we systematically observed that the crack finally occurred in the zone of highest dissipation [36].

A progressive increase in the dissipation intensity per cycle as a function of the number of cycles can also be observed in Fig. $6(a-b)$. From a theoretical standpoint, this property is not compatible with the hypothesis of a stabilized cyclic state often used in the fatigue lifespan criteria. Indeed, in a general case, the hysteresis area is induced by energy storage or release, energy dissipation and thermomechanical coupling mechanisms [37]. This can symbolically be formulated as: 
Fig. 10 2D distribution of intrinsic dissipation at: (a) $0.3 \times 10^{5}$ cycles (b) $0.6 \times 10^{5}$ cycles (c) $10^{6}$ cycles (a)

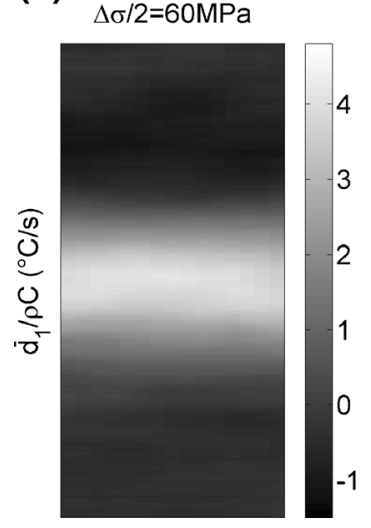

(b)

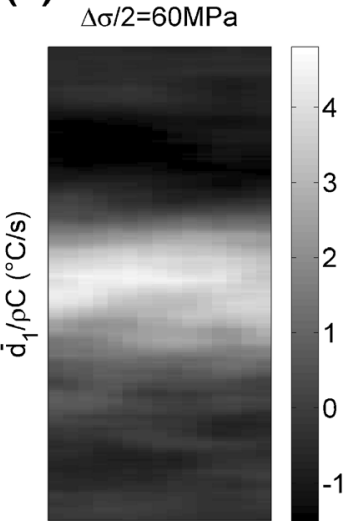

(c) $\Delta \sigma / 2=60 \mathrm{MPa}$

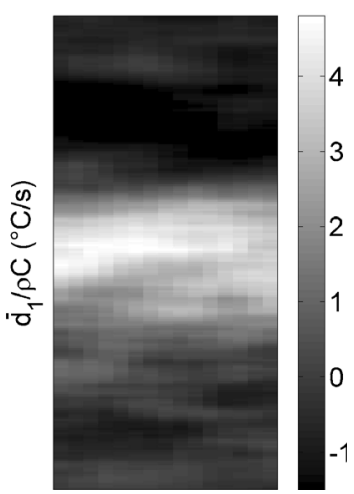

$w_{\text {def }}=\int_{\text {cycle }} \sigma: \mathrm{d} \varepsilon=\int_{\text {cycle }} \rho\left(\mathrm{d} e^{-} \rho C \mathrm{~d} T\right)+\left(d_{1}+s_{\text {thc }}\right) \mathrm{d} t$

where $e$ is the specific internal energy of the material. Here, we recognize that coupling effects (thermoelastic ones) can be neglected over a cycle, with the process being approximately adiabatic. Consequently, only dissipation and energy storage are associated with the hysteresis loop here.

- If there is no energy storage, the dissipation increasing, the hysteresis loop cannot be stabilized and consequently there is no stabilized thermodynamic cyclic state.

- If energy storage exists, the possibility of having a stabilized hysteresis loop may occur; however the mechanical cycle is no longer a thermodynamic cycle, and the cyclic stabilization becomes impossible.

So, even if the dynamic stress-strain response is still currently out of reach from an experimental standpoint, we can conclude that viscoplastic shakedown does not occur.

This energy analysis led us to formulate a possible definition of the fatigue limit associated with an infinite lifespan: this limit could exist if and only if any loading cycle, characterized by a stress range less than the fatigue limit, becomes a thermodynamic cycle. During this cycle, either no intrinsic dissipation occurs or all the deformation energy lost over the cycle is dissipated.

\section{Stress Gradient Effects on Dissipation}

As already mentioned, the dissipation profiles were obtained, in these previous tests, for different stress ranges assessed in the middle of the sample gage part. The dynamic effects in a specimen with variable cross-sections led to heterogeneous stress range patterns (cf Fig. 1). The dissipation profiles allowed us to study the possible influence of this stress range gradient. In Fig. 7, the absolute value of the stress range gradient is plotted as a function of the stress range itself. Each curve corresponds to the distribution of stress range gradients for a given loading condition. These latter are represented by the stress range assessed in $\mathrm{x}=0$, where $\left|\frac{\partial \sigma}{\partial x}\right|=0$ and corresponding to the intersection of each curve with the abscissa axis. In Fig. 8, the intrinsic dissipation curves are plotted as a function of the stress range for different stress range gradient values. These curves are very close to each other, thus indicating a negligible influence of the stress range gradient.
Fig. 11 Mean dissipation vs. stress amplitude (a) full scale, (b) zoom (a)

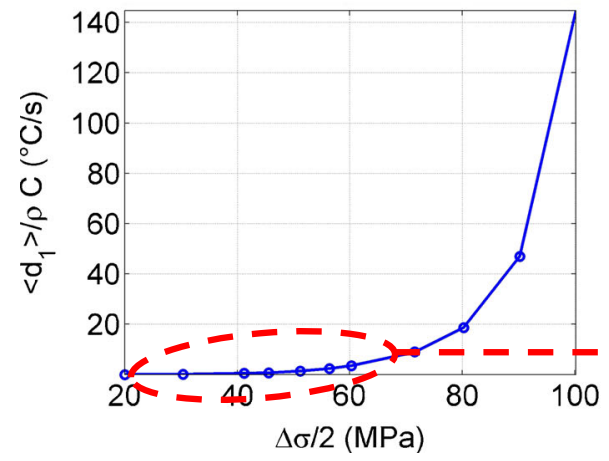

(b)

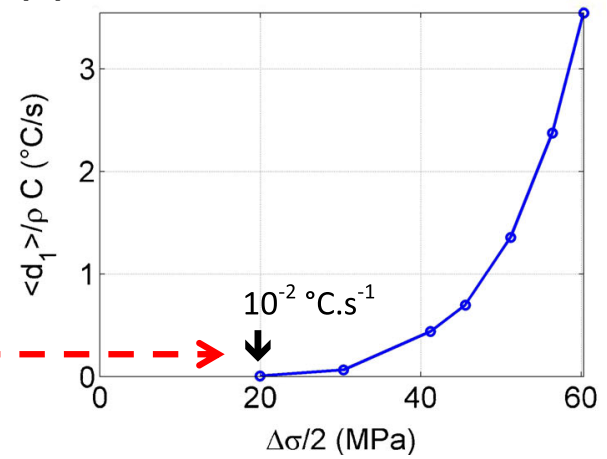




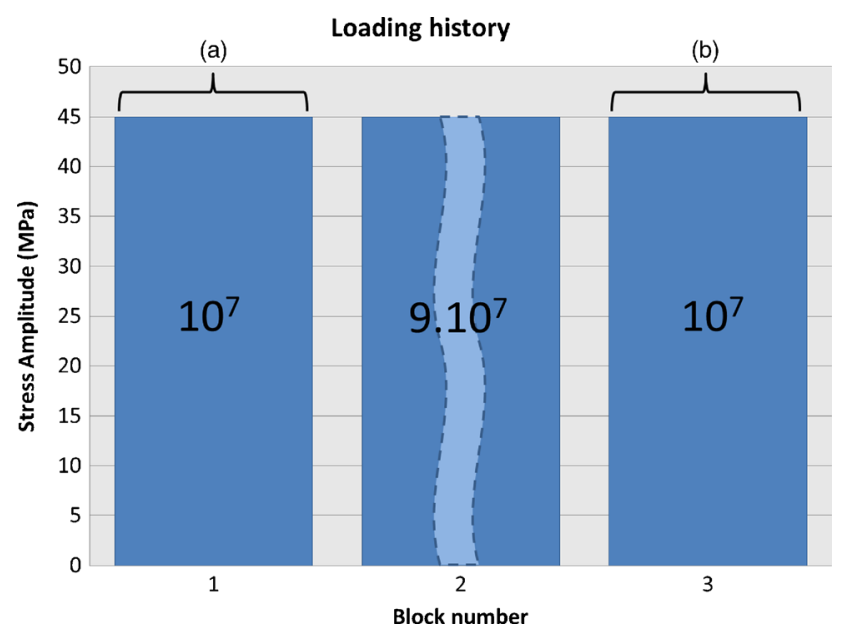

Fig. 12 VHCF fatigue tests: (a) block \#1: first recording period (b) block\#3: second recording period, $\Delta \sigma / 2=45 \mathrm{MPa}$

\section{Spatiotemporal Dissipation Patterns}

Two-dimension dissipation fields can also be computed. These dissipation fields were estimated using the temperature on the specimen surface (black rectangle in Fig. 9a). The abscissa corresponds to the specimen width and the ordinate to the length. The colorbar highlights the extent of self-heating, with the room temperature being around $20{ }^{\circ} \mathrm{C}$.

Examples of 2D dissipation fields corresponding to block \#7 are shown in Fig. 10. A progressive narrowing and intensification of the dissipation zone can be noted. The 2D distributions also show that the profiles plotted in Fig. 6(a-b) are satisfactorily representative, with the dissipation gradients remaining low in the width direction.

Let $<d_{1}>$ now be the mean intrinsic dissipation defined by:

$\left\langle d_{1}\right\rangle=\frac{1}{x_{0}} \int_{-x_{0} / 2}^{x_{0} / 2} \overline{\bar{d}}_{1} \mathrm{~d} x$

This mean value is computed over a zone centered in the middle of the specimen, at the end of each block of cycles. The length $x_{0}$ is here equal to $8 \mathrm{~mm}$ (see Fig. 6). The diagram $<d_{1}>$ vs. $\Delta \sigma$ is plotted in Fig. 11(a-b). We can observe that dissipation occurs regardless of the reachable applied load. The dissipation intensity obtained for the lowest strain amplitude (experimentally reachable) is about $10^{-2}{ }^{\circ} \mathrm{C} . \mathrm{s}^{-1}$ (i.e. $34 \mathrm{~kW} \cdot \mathrm{m}^{-3}$ ). The systematic presence of energy dissipation, as also observed in [38], asks the question of the relevance of the concept of elastic shakedown.

A non-linear increase in mean dissipation can also be observed. This non-linear pattern has already been noted in HCF tests [19, 34, 38-40].

Many different fatigue tests at a higher number of cycles have also been performed. In Fig. 12, the goal is to observe the drift in the thermal and calorimetric signals as a function of the (large) number of cycles at "constant" stress range (here $90 \mathrm{MPa}$ ).

In order to limit data storage, the image recording is only performed during the two "short" blocks (a) and (b), which consists of $10^{7}$ cycles (see Fig. 12). Between both blocks, a block of $9 . \times 10^{7}$ cycles is performed.

Similarly as in equation (12), let us define the mean temperature variation by:

$\langle\theta\rangle=\frac{1}{x_{0}} \int_{-x_{0} / 2}^{x_{0} / 2} \overline{\bar{\theta}} \mathrm{d} x$

In Fig. 13, the results show temperature (a) and dissipation drifts (b) as a function of the number of cycles. The marked difference of magnitude observed between both recordings shows the evolution of the material state during the largest block \#2, which included $9 . \times 10^{7}$ cycles.

\section{Conclusion}

In this work, heat diffusion models are proposed to estimate the dissipated energy during ultrasonic VHCF tests from
Fig. 13 Temperature time course (a) Block \#1 (b) Block \#3, $\Delta \sigma / 2=$ $45 \mathrm{MPa}$ (a)

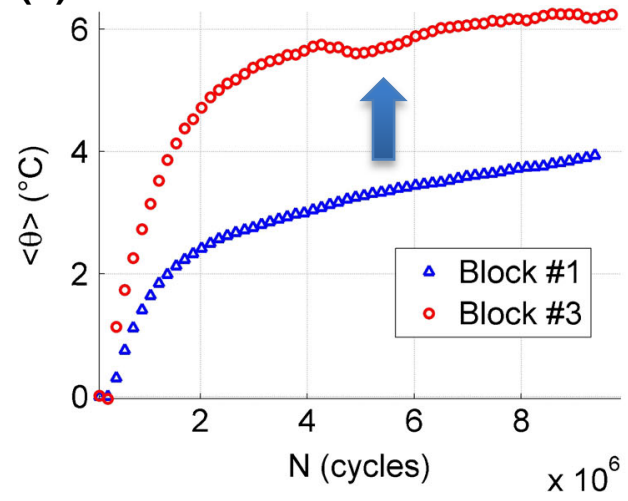

(b)

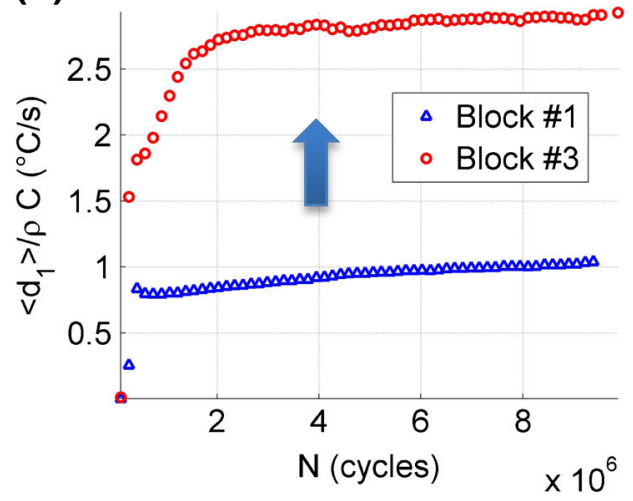


temperature measurements provided by an IR camera. The results showed that:

- Energy dissipation occurs whatever the reachable stress range. Moreover, the dissipation intensity drastically increases with the stress range. No thermodynamic reversible domain can be clearly identified.

- Temperature variation and dissipation intensity systematically grow with the number of cycles under constant loading conditions. This growing is associated with the variation in the microstructural state, so no stabilized cyclic states can be expected.

In conclusion, neither elastic nor plastic shakedown occurred during the VHCF fatigue tests on pure copper. In other words, the results show that the fatigue strength of copper (about $93 \mathrm{MPa}$ at $10^{10}$ cycles [27]) cannot be considered as the fatigue limit from a thermodynamic standpoint.

Regarding the prospects, a crucial next stage should be an experimental analysis of the strain fields. It is indeed crucial, for future modeling work, to know the mechanical loading conditions more precisely without assuming the nature of the material behavior. Here we assumed that tests were stress-controlled, which is not true once microplastic hardening and/or damage occurs. The digital image correlation (DIC) method is currently being extended to dynamic tests.

Another important point to consider is related to the nature of the dissipative effects. Based on experiments on ferritic steel, some theoretical approaches estimate that the change in the self-heating regime is associated with the change in deformation mechanism: from viscoelastic, mechanically recoverable, mechanisms at low stress amplitudes (e.g. anelastic cyclic oscillations of anchored dislocations) to nonrecoverable viscoplastic mechanisms (e.g. inelastic dislocations glide) [38, 41]. The first calorimetric analysis during quasi-static HCF tests [31, 39] revealed an independence of the dissipated energy per cycle as a function of the loading frequency suggesting the occurrence of the same deformation mechanisms over the studied stress amplitude range. Investigating the frequency impact on the dissipation, other works also suggested the occurrence of plastic deformation in both regimes but which is more or less localized at the grain scale [42]. All these results led us to design second horn to operate at $30 \mathrm{kHz}$. Comparing the dissipation levels at 20 and $30 \mathrm{kHz}$ should allow us to better understand the dissipative mechanisms in dynamic VHCF.

Acknowledgments The authors wish to thank the Agence Nationale de la Recherche France ANR-09-BLAN-0025-01 for its financial support in the framework of the ANR project DISFAT.

\section{References}

1. Bathias C (1999) There is no infinite fatigue life in metallic materials. Fatigue Fract Eng Mater Struct 22(7):559-565

2. Pyttel B, Schwerdt D, Berger C (2011) Very high cycle fatigue-Is there a fatigue limit? Int J Fatigue 33(1):49-58

3. Bathias C, Drouillac L, Le François P (2001) How and why the fatigue $\mathrm{S}-\mathrm{N}$ curve does not approach a horizontal asymptote. Int $\mathrm{J}$ Fatigue 23(1):143-151

4. Stanzl-Tschegg S (2013) Very high cycle fatigue measuring techniques. Int J Fatigue. doi:10.1016/j.ijfatigue.2012.11.016

5. Ebara R (2006) The present situation and future problems in ultrasonic fatigue testing - Mainly reviewed on environmental effects and materials' screening. Int J Fatigue 28(11):1465-1470

6. Laird HM (1995) Frequency effects on cyclic plastic strain of polycrystalline copper under variable loading. Mater Sci Eng A 194(2): $137-145$

7. Morrissey RJ, Golden PJ (2007) Fatigue strength of a single crystal in the gigacycle regime. Int J Fatigue 29(9-11):2079-2084

8. Morrissey RJ, McDowell DL, Nicholas T (1999) Frequency and stress ratio effects in high cycle fatigue of Ti-6Al-4V. Int J Fatigue 21(7):679-685

9. Fatemi A, Yang L (1998) Cumulative fatigue damage and life prediction theories: a survey of the state of the art for homogeneous materials. Int J Fatigue 20(1):9-34

10. Ozaltun H, Shen M-H, George T, Cross C (2011) An energy based fatigue life prediction framework for in-service structural components. Exp Mech 51(5):707-718

11. Kim J, Jeong H-Y (2010) A study on the hysteresis, surface temperature change and fatigue life of SM490A, SM490A-weld and FC250 metal materials. Int J Fatigue 32(7):1159-1166

12. Li H, Nishimura A, Nagasaka T, Muroga T (2007) Fatigue life and cyclic softening behavior of JLF-1 steel. Fusion Eng Des 82(15-24): 2595-2600

13. Amiri M, Khonsari M (2010) Rapid determination of fatigue failure based on temperature evolution: fully reversed bending load. Int $\mathrm{J}$ Fatigue 32(2):382-389

14. Doudard C, Calloch S, Cugy P, Galtier A, Hild F (2005) A probabilistic two-scale model for high cycle fatigue life predictions. Fatigue Fract Eng Mater Struct 28:279-288

15. Ezanno A, Doudard C, Calloch S, Millot T, Heuzé J-L (2010) Fast characterization of high-cycle fatigue properties of a cast copperaluminum alloy by self-heating measurements under cyclic loadings. Procedia Eng 2(1):967-976

16. Krapez J-C, Pacou D (2002) Thermography detection of early thermal effects during fatigue tests of steel and aluminum samples. Am Inst Phys Conf Proc 615(1):1545-1552

17. La Rosa G, Risitano A (2000) Thermographic methodology for rapid determination of the fatigue limit of materials and mechanical components. Int J Fatigue 22(1):65-73

18. Le Saux V, Marco Y, Calloch S, Doudard C, Charrier P (2010) An energetic criterion for the fatigue of rubbers: an approach based on a heat build-up protocol and $\mu$-tomography measurements. Procedia Eng 2(1):949-958

19. Poncelet M, Doudard C, Calloch S, Weber B, Hild F (2010) Probabilistic multiscale models and measurements of self-heating under multiaxial high cycle fatigue. J Mech Phys Solids 58:578-593

20. Luong MP (1998) Fatigue limit evaluation of metals using an infrared thermographic technique. Mech Mater 28(1-4):155-163

21. Luong MP (1995) Infrared thermographic scanning of fatigue in metals. Nucl Eng Des 158(2-3):363-376

22. Galtier A, Bouaziz O, Lambert A (2002) Influence de la microstructure des aciers sur leur propriétés. Méc Ind 3(5):457-462

23. Mason W (1950) Piezoelectric crystals and their application to ultrasonics. Van Nostrand, New York 
24. Bathias C (2006) Piezoelectric fatigue testing machines and devices. Int J Fatigue 28(11):1438-1445

25. Bathias C, Paris P, Dekker M (eds) (2004) Gigacycle fatigue in mechanical practice. CRC Press, Boca Ranton

26. Papadopoulos IV, Panoskaltsis VP (1996) Invariant formulation of a gradient dependant multiaxial high-cycle fatigue criterion. Eng Fract Mech 55(4):513-528

27. Stanzl-Tschegg S, Mughrabi H, Schoenbauer B (2007) Life time and cyclic slip of copper in the VHCF regime. Int J Fatigue 29(9-11): 2050-2059

28. Phung NL (2012) Fatigue sous très faibles amplitudes de contrainte : Analyse des mécanismes précurseurs de l'amorçage de fissure dans le cuivre polycristallin. PhD Thesis, ENSAM Paris

29. Honorat V, Moreau S, Muracciole J, Wattrisse B, Chrysochoos A (2005) Calorimetric analysis of polymer behaviour using a pixel calibration of an IRFPA camera. Quant InfraRed Thermography J 2:153-171

30. Germain P, Nguyen QS, Suquet P (1983) Continuum Thermomechanics. J Appl Mech Trans ASME 50(4B):1010-1020

31. Berthel B, Chrysochoos A, Wattrisse B, Galtier A (2008) Infrared image processing for the calorimetric analysis of fatigue phenomena. Exp Mech 48:79-90

32. Boulanger T, Chrysochoos A, Mabru C, Galtier A (2004) Calorimetric analysis of dissipative and thermoelastic effects associated with the fatigue behavior of steels. Int J Fatigue 26(3):221-229

33. Chrysochoos A, Louche $H$ (2000) An infrared image processing to analyse the calorific effects accompanying strain localisation. Int $\mathrm{J}$ Eng Sci 38(16):1759-1788

34. Mareau C, Favier V, Weber B, Galtier A (2009) Influence of the free surface and the mean stress on the heat dissipation in steels under cyclic loading. Int J Fatigue 31(8-9):1407-1412
35. Doudard C, Calloch S, Hild F, Roux S (2010) Identification of heat source fields from infra-red thermography: determination of 'selfheating' in a dual-phase steel by using a dog bone sample. Mech Mater 42:55-62

36. Wang C, Blanche A, Wagner D, Chrysochoos A, Bathias C (2014) Dissipative and microstructural effects associated with fatigue crack initiation on an Armco iron. Int J Fatigue 58:152 157

37. Chrysochoos A, Huon V, Jourdan F, Muracciole J-M, Peyroux $\mathrm{R}$, Wattrisse B (2010) Use of full-field digital image correlation and infrared thermography measurements for the thermomechanical analysis of material behaviour. Strain 46(1):117-130

38. Connesson N, Maquin F, Pierron F (2011) Dissipated energy measurements as a marker of microstructural evolution: $316 \mathrm{~L}$ and DP600. Acta Mater 59(10):4100-4115

39. Berthel B, Wattrisse B, Chrysochoos A, Galtier A (2007) Thermographic analysis of fatigue dissipation properties of steel sheets. Strain 43(3):273-279

40. Morabito A, Chrysochoos A, Dattoma V, Galietti U (2007) Analysis of heat sources accompanying the fatigue of 2024 T3 aluminium alloys. Int J Fatigue 29(5):977-984

41. Mareau C, Favier V, Weber B, Galtier A, Berveiller M (2012) Micromechanical modeling of the interactions between the microstructure and the dissipative deformation mechanisms in steels under cyclic loading. Int J Plast 32-33:106-120

42. Munier R (2012) Etude de la fatigue des aciers laminés à partir de l'auto-échauffement sous sollicitation cyclique: Essais, observations, modélisation et influence d'une pré-déformation plastique. $\mathrm{PhD}$ thesis, Université de Bretagne Occidentale 